Nouvelles perspectives en sciences sociales

Revue internationale de systémique complexe et d'études relationnelles

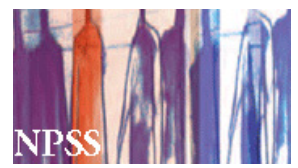

\title{
Cycle de la négation en arabe marocain
}

\section{Nizha Chatar-Moumni}

Volume 9, numéro 1, novembre 2013

Sur le thème : linguistique et complexité

URI : https://id.erudit.org/iderudit/1024043ar

DOI : https://doi.org/10.7202/1024043ar

Aller au sommaire du numéro

\section{Éditeur(s)}

Prise de parole

\section{ISSN}

1712-8307 (imprimé)

1918-7475 (numérique)

Découvrir la revue

\section{Citer cet article}

Chatar-Moumni, N. (2013). Cycle de la négation en arabe marocain. Nouvelles perspectives en sciences sociales, 9(1), 219-238.

https://doi.org/10.7202/1024043ar

\section{Résumé de l'article}

En arabe marocain, la négation grammaticale est réalisée soit par le seul élément ma- soit par l'association de ma- à l'élément -š ou sa variante -ši. L'analyse des relations qu'entretiennent ces deux éléments nécessite de prendre conjointement en compte différents niveaux d'analyse : la morphologie, la syntaxe, la sémantique, la lexicologie, la pragmatique, mais aussi la logique. L'auteur explore les racines historiques de la négation en arabe afin de saisir les facteurs conditionnant l'usage en synchronie de la négation en arabe marocain défend l'hypothèse que l'unité ma- doit nécessairement être associée à un quantifieur véhiculant un trait de sens [+ non-défini]. L'association avec -š est liée à la présence ou l'absence de ce trait de sens, mais aussi à la force pragmatique de la négation. 


\title{
Cycle de la négation en arabe marocain
}

\author{
Nizha Chatar-Moumni \\ Université Paris Descartes, \\ MoDyCo, UMR 7114
}

\begin{abstract}
$\mathbf{O}$
$\mathrm{n}$ assiste actuellement à la résurgence du débat sur la notion de "complexité » appliquée aux langues et au langage ${ }^{1}$ : les langues sont-elles de complexité équivalente ${ }^{2}$ ou bien présententelles des degrés de complexité? Les travaux défendant la seconde thèse distinguent généralement deux types de complexité linguistique : la complexité absolue - orientée vers une théorie qui mesure et compare le degré de complexité de chaque constituant grammatical, et la complexité relative - orientée vers l'usager - qui évalue et compare les difficultés du processus d'acquisition-apprentissage des langues ${ }^{3}$. Dans cet article, nous nous inscrivons dans le premier de ces points de vue tout en retenant l'idée formulée par Rescher que "Any sort of system or process - anything that is structured whole consisting of interrelated
\end{abstract}

\footnotetext{
$\overline{1}$ Le titre de cet article fait référence au « cycle de Jespersen ", voir le point 2 (Origine de la négation) ci-dessous.

2 "C'est une découverte de la linguistique moderne, que toutes les langues sont à peu près égales en termes de complexité générale ". Robert Dixon, The Rise and Fall of Languages, Cambridge, Cambridge University Press, 1997, p. 118.

3 Voir Matti Mietsamo, "On the Complexity of Standard Negation ", dans Mickael Suominen et al. (dir.), A Man of Measure: Festschrift in Honour of Fred Karlsson on his 60th Birthday, Turku, Linguistic Association of Finland, 2006, p. 346.
} 
parts - will be to some extent complex " ». En particulier, il s'agira ici d'observer et d'analyser les relations mises en jeu dans la construction du sens par la négation grammaticale en arabe marocain (désormais AM). En AM, la négation est réalisée soit par le seul élément $m a$ - soit par l'association de $m a$-à l'élément $-s$ ou sa variante $-\check{s} i$. L'analyse des relations qu'entretiennent ces deux éléments nécessite de prendre conjointement en compte différents niveaux d'analyse : la morphologie, la syntaxe, la sémantique, la lexicologie, la pragmatique, mais aussi la logique. "Concept primitif et universel, la négation met en jeu toutes les sciences du langage. En effet, pour tenter de comprendre sa fonction il faudrait avoir une conception globale de l'activité linguistique ${ }^{5}$ ".

L'AM est une langue du groupe sémitique de la famille des langues chamito-sémitiques (ou afro-asiatiques). C'est une langue à tradition orale utilisée dans les situations de communication les plus diversement informelles ${ }^{6}$. L'AM coexiste, entre autres, avec l'arabe standard moderne, langue écrite, généralement acquise en milieu scolaire ${ }^{7}$, et utilisée dans des situations très formelles. La variété d'AM parlée par nos informateurs est celle d'Oujda, ville située au nord-est du Maroc et comptant plus d'un million d'habitants.

Cet article est articulé comme suit. Nous commencerons par identifier les contextes qui conditionnent en synchronie les formes de la négation en AM. Nous donnerons ensuite un bref historique de sa genèse. Ceci nous permettra, d'une part, de mettre en évidence d'intéressantes analogies entre le français et l'AM et, d'autre part, de souligner l'importance des concepts de " portée " et de " quantification " pour la compréhension des

$4 \quad$ Nicolas Reschler, Complexity: A Philosophical Overview, New Jersey, Transaction publishers, 1998, p. 1.

5 Henning Nølke, "Les lectures de ne... pas : éléments d'une explication modulaire ", Linx [En ligne], 5, 1994, consulté le 25 octobre 2012, http:// linx.revues.org/1212, DOI : 10.4000/linx.1212.

6 L'arabe marocain commence toutefois à apparaître sous une forme écrite, notamment sur Internet, dans les sms et la publicité.

7 Le français, autre langue dominant le paysage linguistique marocain, est enseigné à partir de la troisième année primaire. 
différentes " associations négatives ${ }^{8}$ " entre $m a$ - et des termes à polarité négative (TPN). Nous nous arrêterons enfin sur un TPN en particulier, ћetta " même; jusqu'à ", afin d'illustrer la complexité de traitement et d'interprétation de certains énoncés négatifs.

\section{Formes de la négation}

La négation grammaticale en AM peut être marquée par les deux éléments $m a$ - et $-\check{s}$ ou sa variante $-s ̌ i$ :
(1) l-mra
$m a-z ̌ a-t-s ̌ s$
l-l-eers
la-femme nég ${ }^{9}$-venir-elle.perf-q à-le-mariage

'La femme n'est pas venue au mariage.'

(2) $\boldsymbol{m a} \boldsymbol{a}-\varepsilon n d-h a-{ }^{-}$

nég-chez-elle-q

l-flus

'Elle n'a pas d'argent.'

(3) l-mra

ma-ši $f$-l-eers

la-femme nég-q dans-le-mariage

'La femme n'est pas au mariage.'

Notons que dans d'autres variétés d'arabe parlé - pour le même type de contexte - ma- est utilisé seul. C'est le cas, par exemple, en arabe soudanais (4, ci-dessous) ou encore en hassania (5) parlé en Mauritanie et dans la région de Tan-Tan au Maroc. Dans d'autres variétés, en revanche, on trouvera le seul $-\check{s}$, comme en arabe libanais (6) ou en arabe jordanien $(7)^{10}$ :

(4)
Omar
$m a-d \check{z} a$
la-femme nég-venir.il.perf
'Omar n'est pas venu.'

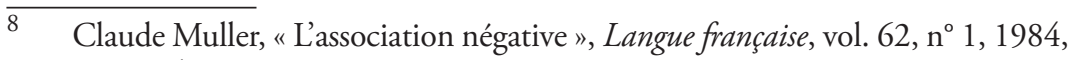
p. 59-94.

9 Abréviations : acc : accusatif; déf : défini; $\mathrm{f}$ : féminin; imp : imperfectif; indef : indéfini; lit. : littéralement; $\mathrm{m}$ : masculin; nég : négation; perf : perfectif; $\mathrm{pl}$ : pluriel; q : quantifieur; TPN : terme à polarité négative

10 Ces exemples sont empruntés à Elabbas Benmamoun, The Feature Structure of Functional Categories. A Comparative Study of Arabic Dialects, New-York/ Oxford, Oxford University press, 2000, p. 70. 
(5) maa-št $y a l-t$ nég-travailler-je.perf 'Je n'ai pas travaillé.'

(6) $b i-t-\hbar i b-s$ sizl il-bayt asp.elle.imp.aime-nég travail la-maison 'Elle n'aime pas le ménage.'

(7) beddi-šs veux-moi-nég 'Je ne veux pas.'

En AM, le système est plus complexe. D’une part, ma- et $-\check{s} /-s ̌ s i$ peuvent être séparés ou réunis selon les contextes. Dans des assertions directes, $m a-$ et $-\check{s}$ entourent un prédicat verbal (1) ou quasi-verbal (2) mais précèdent ( $m a-s i$ i $)$ un prédicat non-verbal (3). Dans des énoncés polémiques ou adversatifs, c'est-à-dire des énoncés marqués par le locuteur, on trouvera l'inverse; en (8) $m a-s ̌ i$ précèdent un prédicat verbal, mais entourent un prédicat non verbal en (9):

(8) $\hbar m e d ~ k l a-\emptyset$ ?

Ahmed manger-il.perf

'Ahmed a mangé?'

ma-ši kla-ø! Eammer-ø-ha!

nég-q manger-il.perf remplir-il.perf-elle

'Il n'a pas mangé! Il s'est empiffré!

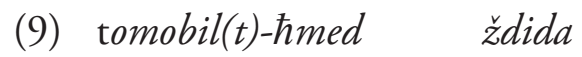
voiture-Ahmed neuve

'La voiture de Ahmed est neuve.'

ma-ždida-šs! Eend-ha Eam-in

nég-neuve-q chez-elle année-duel

'Elle n'est pas neuve! Elle a deux ans.'

Dans le contexte d'un nom non déterminé (10), dans les serments (11) ou encore dans le contexte d'une relative introduite par la variante $m a$ du relatif $(12)^{11}$, la négation est réalisée par le seul ma- :

$\overline{11}$ Pour plus de détails, nous renvoyons le lecteur à, entre autres, Elabbas 
(10) $\boldsymbol{m a} \boldsymbol{a}-\varepsilon e n d-i \quad f$ fus

nég-à-moi argent

'Je n'ai pas d'argent.'

(11) wallah

$m a-n-z ̌ i-h a$

par Dieu nég-je.imp-venir-elle

'Je jure de ne pas venir.'

(12) $m a-\varepsilon e n d-u \quad m a-i-g u l$

nég-chez-lui quoi-il.imp-dire

'Il n'a rien à dire

La question se pose alors des facteurs qui conditionnent l'association de $m a-$ et- $r$. Dans cet article, nous restreindrons notre observation aux contextes dans lesquels la négation détermine un prédicat verbal ou quasi-verbal.

\section{Origine de la négation}

La négation est un des thèmes majeurs des recherches dans le domaine de la grammaticalisation. Il semblerait d'ailleurs que le terme même de grammaticalisation ait été utilisé la première fois par Meillet ${ }^{12}$ pour expliquer, parmi d'autres faits linguistiques, l'évolution de la négation du latin au français. On doit à

Benmamoun, "Licensing of Polarity Items in Moroccan Arabic ", Natural Language and Linguistics Theory, $\mathrm{n}^{\circ}$ 15, 1997, p. 263-287; Elabbas Benmamoun, "Licensing Configurations: The Puzzle of Head Negative Polarity Items ", Linguistic Inquiry, vol. 33, n 1, 2006, p. 141-149; Kristen Brustad, The Syntax of Spoken Arabic. A Comparative Study of Moroccan, Egyptian, Syrian, and Kuwaiti Dialects, Washington, D.C., Georgetown University Press, 2000; Dominique Caubet et Salem Chaker (dir.), La négation en berbère et en arabe maghrébin, Paris, L'Harmattan, 1996; Nizha Chatar-Moumni, "Quelques aspects du fonctionnement de la négation en arabe marocain ", La linguistique, vol. 44, n 2, 2008, p. 81-97; Richard Slade Harrell, A Short Reference Grammar of Moroccan Arabic, Washington DC, Georgetown University Press, 1962; Frederick Hoyt, "Sentential Negation Marking in Palestinian and Moroccan Arabic. A Study in Comparative Syntax ", Seminar of Arabic Dialectology, http://fmhoyt.colliertech.org/ Hoyt(05)_NegationInTwoDialectsOfArabic.pdf (consulté le 15/11/2013), 2005; Philippe Marçais, Le parler arabe de Djidjelli, Paris, Maisonneuve, 1953.

12 Antoine Meillet, "L'évolution des formes grammaticales ", Scientia. Revue internationale de synthèse scientifique, vol. 12, n XXVI-6, 1912, p. 384-400. 
Jespersen ${ }^{13}$ d'avoir développé et expliqué le processus du changement syntaxique de la négation. Ce processus a été nommé bien plus tard par Dahl ${ }^{14}$, le " cycle de Jespersen " ou " cycle de la négation ». Le français et l'arabe présentent des similarités dans le processus de renouvellement de la négation plutôt évidentes; nous les esquisserons ici.

2.1. Le français ne... pas

La négation grammaticale du français vient du latin non, unité préverbale (13), pouvant être renforcée sémantiquement par des unités du type de micam 'mie' (14) :

(13) difficile est saturam non scribere

'Il est difficile de ne pas écrire de satire.'

(Juvénal)

(14) non micam mentis sanae habere

Lit. : pas une miette d'esprit sain avoir.' 'Être complètement fou'

En vieux français, non, phonétiquement réduit et non accentué, a évolué en ne (15) et s'est associé à des unités dénotant la plus petite quantité possible dans un domaine d'expérience ${ }^{15}$ (pas, miette, goutte, point) en fonction, notamment, de la classe sémantique du prédicat verbal : pas avec des verbes de mouvement, goutte avec des verbes du type boire, miette avec des verbes du type de manger, etc.

\section{Quel part quil alt, ne poet mie cäir}

(Chanson de Roland, 2034, TL6, 15, 23)

Lit. : où qu'il aille, il ne peut une miette tomber (= un peu)

13 Otto Jespersen, « Negation in English and Other Languages », Copenhagen, Høst, 1917.

14 Östen Dahl, « Typology of Sentence Negation », Linguistics, vol. 17, 1979, p. 79-106.

15 Michèle Fruyt, "Négation et grammaticalisation en latin ", Revue de linguistique latine du centre Alfred Ernout : de lingua latina, $\mathrm{n}^{\circ} 1,2008$, (revue électronique : http://www.fabula.org/actualites/de-lingua-latina-revue-delinguistique-latine-du-centre-alfred-ernout_28051.php), article consultable à : http://www.paris-sorbonne.fr/IMG/pdf/Fruyt2.pdf (consulté le 12 $15 / 11 / 2013)$. 
'Où qu'il aille, il ne peut point tomber.'

Ces unités se sont progressivement vidées de leur poids sémantique (lexical) pour endosser un poids grammatical par " contamination » avec ne. Les possibilités se sont ensuite réduites une à une à une en faveur de l'association de ne avec point (16) dans les registres formels et pas dans les registres informels (17). Aujourd'hui, on le sait, dans une troisième étape du processus du " cycle de la négation ", pas peut être utilisé seul à l'oral (18) : (16) Il ne mange point.

(17) Il ne mange pas.

(18) Il mange pas.

\subsection{L'arabe marocain $m a-s ̌$}

L'unité ma-dérive probablement de l'arabe classique qui marque la négation syntaxique par une unité simple préverbale : laa, lam, lan, maa ou laysa en fonction des modalités temporelles et aspectuelles du verbe et du type de phrase. Quant à $-s /-s ̌ i$, il nous vient le plus vraisemblablement de l'arabe classique šay'an 'une chose', c'est-à-dire le nom šay' à l'accusatif, tel qu'on peut le trouver dans les exemples extraits du Coran (19) et (20) ci-dessous. Dans ces deux exemples, šay' sert à renforcer sémantiquement, à emphatiser la négation laa. En (19), l'association donne littéralement le sens de "pas une chose ", c'est-à-dire "rien "; en (20), la même association signifie en revanche littéralement " pas en une chose ", c'est-à-dire " en rien ».
(19) wa là
$t-u s ̌ r i k-\bar{u}$
$b i-h i$
$\stackrel{s}{s} a y^{\prime}-a n^{16}$

$\overline{16}$ Voir Mostafa Lameen Souag, Explorations in the Syntactic Cartography of Algerian Arabic, thèse SOAS, London University, 2006; Christopher Lucas, "Jespersen's cycle in Arabic and Berber ", Transactions of the Philological Society, Cambridge, Cambridge University, vol. 105, n 3, 2007, p. 398-431; Nizha Chatar-Moumni, 2008, op. cit.; Nizha Chatar-Moumni, " Negation in Moroccan Arabic: Scope and Focus ", dans Reem Bassiouney et Graham Katz (dir.), Arabic Language and Linguistics, Georgetown, Georgetown University Press, 2012, p. 3-15; Christopher Lucas et Elliott Lash, "Contact as Catalyst: The Case for Coptic Influence in the Development of Arabic Negation ", Journal of Linguistics, $\mathrm{n}^{\circ}$ 46, 2010, p. 379-413. 
et nég 2.imp-associer-m.pl avec-Lui chose-acc Lit. : et n'associez pas avec lui une chose. 'Et ne Lui associez rien.'

(Coran, 4:36)

(20) inna Allāha lā ya-ðlim-u ñà̃s-a šaý-an certes Allah nég il.imp-être injuste les-gens-acc chose-acc Lit. : certes Allah n'est pas injuste avec les gens en rien 'En vérité, Allah n'est point injuste à l'égard des gens.'

(Coran, 10:44)

Il est intéressant de noter que dans l'exemple (19) le nom šay' échappe au contrôle de la négation (19' est tout à fait possible), ce qui n'est pas le cas dans (20); (20’) ci-dessous n'est pas grammatical :

(19') wa t-ušrik-ù bi-hi šay-an (20') *Allāh ja Xlim-u n-naas-a šay'-an

En (20), la négation fonctionne comme une unité à portée dans le sens où elle exerce " un contrôle structurel sur un fragment de la phrase ${ }^{17}$ ». Selon Lucas et $\mathrm{Lash}^{18}$, šay" "is found predominantly in the context of negation already in Classical Arabic. In the Qur'an, for example, which consists of approximatively 80,000 words, say? an occurs 77 times. Of these, fully 63 (81,8\%) occur in the scope of negation ". Il semble donc que la fréquence élevée de šaýan "chose » dans le contexte de la négation l'a progressivement rendue sensible à la négation et en a fait un terme à polarité négative (TPN), c'est-à-dire un terme ne pouvant apparaître que dans le contexte de la négation.

En français, comme en arabe, les domaines de la négation et de la quantification sont étroitement liés. Mais si en français, la négation s'est renouvelée via un minimizer, c'est-à-dire un terme dénotant la plus petite entité possible dans un domaine donné de l'expérience (pas, goutte, mie, point), en arabe, elle s'est associée à une unité dénotant une quantité vague, indéterminée ou encore

$17 \quad$ Henning Nølke, Linguistique modulaire : de la forme au sens, Louvain-Paris, Peeters, 1994 , p. 228.

18 Christopher Lucas et Elliott Lash, op. cit., p. 382. 
indéfinie. Cette quantité - très petite ou très vague - s'est réduite au contact de la négation à une quantité nulle.

Selon Claude Muller, la négation syntaxique du français résulte de l'association entre la négation et un quantifieur, ce qu'il nomme «l'association négative " :

Il est bien connu que la négation implique une vision totale du domaine de quantification; pour dire : Il y a quelqu'un dans l'assistance qui est chauve, il n'est pas nécessaire de voir tout le monde. Cela est nécessaire pour pouvoir dire : Il n'y a personne dans l'assistance qui soit chauve. Ce pourrait être l'origine de la présence de quantifieurs en ancien français comme pas, mie, goutte, brin, point, sur lesquels porte la négation pour signifier que l'ensemble du domaine a été pris en considération ${ }^{19}$.

En nous appuyant sur Muller, nous défendrons ici l'hypothèse que, pour satisfaire l'association négative en AM, la négation ma- doit nécessairement être associée à un quantifieur - noté ici $q$ - véhiculant un trait de sens [+ non-défini]. La réalisation de l'unité $-s ̌$ est liée à la présence ou l'absence de ce trait de sens.

\section{Associations négatives en AM}

L'association négative standard en AM lie bien sûr $m a$ - et la forme phonétiquement réduite et sémantiquement faible de l'AC šay’an, le quantifieur indéterminé et non marqué $-\check{s}$ :

ma-kla-ø-šr mea-na lyum
nég-manger-il.perf-q avec-nous aujourd'hui
'Il n'a pas mangé avec nous aujourd'hui.'

Mais ma-a également attiré sous sa portée d'autres unités dénotant une quantité indéfinie afin de couvrir les différents domaines de l'expérience. Par exemple, pour le trait [+humain], ma-s'est associée à $\hbar e d d$ de l'arabe classique 'ahad 'un', le plus petit numéral quantifieur, pour signifier " pas un ", c'est-à-dire une quantité zéro : "Aucun mot n'est plus apte à exprimer la valeur indéfinie que le mot qui dénote l'unité : la notion un exemplaire pris entre plusieurs est en effet très proche parente de la notion un exemplaire non identifié ${ }^{20}$ ".

\footnotetext{
19 Claude Muller, 1984, op. cit., p. 94, note 2.

20 Philippe Marçais, op. cit., p. 399.
} 
(22)
$m a-\stackrel{s}{a} a f-\emptyset-n i$
hedd lyum
nég-voir-il.perf-moi un aujourd'hui
'Personne ne m'a vu aujourd'hui.'

Les unités qui s'associent à $m a$ - occupent la position linéaire requise par la fonction syntaxique qu'ils assument : $\hbar e d d$ est sujet en (22) et objet en (23) :

(23) $m a-\check{s} a f-t$

ћedd lyum

nég-voir-je.perf un aujourd'hui

'Je n'ai vu personne aujourd'hui.'

Pour le trait [-humain], l'association lie ma- et walu, wa-lu, qui semble dérivé de l'arabe classique wa-law 'et si', " même si ", qui sert à dénoter l'irréel, l'absence, c'est-à-dire quelque chose de non défini :
ma-kla-
walu
nég-manger-il.perf rien
'Il n'a rien mangé.'

Pour le trait [+ temps] ma- s'est associé à l'unité cemmer - qui nous vient d'une unité signifiant " durée de vie, vie, âge » - pour dire jamais. On peut le comparer au TPN français de ma vie (25) qui ne peut, par ailleurs, coexister avec pas (25') :

(25) De ma vie, je n'ai vu pareille chose!

(25’ *De ma vie, je n'ai pas vu pareille chose!

Notons l'accord entre le possessif de première personne ma et le pronom je. L'AM eemmer est vraisemblablement issu de la grammaticalisation d'un état construit ( $\succsim ð a \bar{f} f a$ ) qui lie dans une relation 'génitive' dénotant l'appartenance le nom eemmer 'vie' et un nominal; un pronom (26) ou un nom (27). Notons ici aussi l'accord entre le nominal et le sujet ${ }^{21}$ :
(26) eemmer-ha ma-ža-t
l-d-dar
jamais-elle nég-venir-elle.perf à-déf-maison
'Elle n'est jamais venue à la maison.'

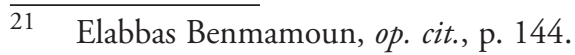


(27)
Eemmer-meryem ma-ža-t
l-d-dar
jamais-Meryem nég-venir-elle.perf à-déf-maison
'Meryem n'est jamais venue à la maison

Eemmer peut précéder ma- (26) ou le suivre (28):

(28)
ma-Eemmer-ha ža-t l-d-dar
nég-jamais-elle venir-elle.perf à-déf-maison
'Elle n'est jamais venue à la maison.'

Mais il ne peut jamais se trouver dans la partie droite de la phrase :

\section{(28') *ma-ža-t eemmer-ha l-d-dar \\ $(28 ”) * m a-\check{z} a-t \quad l-d$-dar eemmer-ha}

La similarité entre le français de ma vie et l'AM Eemmer(-ni), littéralement 'vie(-moi)' est assez intéressante. Tous deux semblent, en effet, fonctionner comme des adverbiaux de durée en position de topique, dans le cas d'une thématisation à l'initiale de l'énoncé. Ceci nous a incité à observer d'autres adverbiaux de durée en AM. Par exemple, hadi šahrin 'voici deux mois' (29) ou telt yam 'trois jours' (30). Ces adverbiaux sont, à l'usage ${ }^{22}$, le plus souvent utilisés à l'initiale de la phrase et, dans cette position, ils entraînent (sans que cela soit obligatoire) la réalisation du seul ma- :
(29) hadi šahr-in
$m a-\check{z} a-\emptyset$
l-d-dar
voici mois-duel nég-venir-il.perf à-la-maison
'Voici deux mois qu'il n'est pas venu à la maison.'
telt yam
$m a-\check{z} a-\emptyset$
$l-d-d a r$
trois jour.pl
nég-venir-il.perf
à-la-maison
'Cela fait trois jours qu'il n'est pas venu à la maison.'

Ils sont généralement peu utilisés à la finale, mais, lorsqu'ils le sont, on constate que l'association entre $m a$-et $-\check{s}$ est obligatoire :

(31) $m a-z ̌ a-\emptyset-\check{s}$

$l-d-d a r$

nég-venir-il.perf-q à-la-maison voici mois-duel

'Cela fait deux mois qu'il n'est pas venu à la maison.'

22 Nous avons testé ces exemples auprès de cinq informateurs natifs de la langue arabe marocain. 
Ces adverbiaux sont, en fait, mis en valeur dans une structure pseudo-clivée que l'on peut d'ailleurs marquer comme telle :
hadi šahr-in
lli $\quad m a-\check{z} a-\emptyset-\check{s}$
$l-d-d a r$
voici mois-duel que nég-venir-il.perf-q à-la-maison
'Voici deux mois qu'il n'est pas venu à la maison.'

L'unité lli, héritée de l'arabe classique al "défini ", porte un trait de sens [+ défini] et de ce fait exige la présence du [-défini] $-\check{s}$ afin de satisfaire " l'association négative». On notera que, placé à l'initiale de la phrase, l'adverbial hadi šahrin contrôle, porte sur la phrase tout entière, assumant, dans cette position, un rôle prédicatif, un rôle de noyau syntaxique. L'AM عemmer, issu de la grammaticalisation d'un adverbial de durée et régulièrement mis en valeur à l'initiale dans les contextes négatifs, s'est cristallisé dans cette position. eemmer dénote une durée indéterminée (durée de vie) qui, au contact de la négation $m a-$, s'est réduit à une quantité zéro.

L'AM ma- a donc attiré sous sa portée différentes unités dénotant toutes une quantité vague, indéterminée, non définie qui, au contact de ma-, s'est réduite à une quantité zéro. Régulièrement sous la portée de la négation, ces quantifieurs sont devenus des TPN en distribution complémentaire.

Dans le paragraphe qui suit, on s'intéressera à une association négative complexe qui lie ma- à l'unité hetta 'même, jusqu'à', le plus souvent identifiée comme un TPN.

\subsection{Association de la structure $\hbar e t t a+\mathrm{N}(\mathrm{om})$ à $m a-$}

La structure de l'AM $\hbar e t t a+N(o m)$ est généralement analysée comme un TPN dans des contextes du type de (33) ci-dessous empruntés à Benmamoun. Notons que hetta est traduit par l'anglais 'any': “One intriguing aspect of NPIs such as ћetta $+N P$ is their complementary distribution with suffix $\check{s}$, as shown in $(\ldots)^{23}$ :

(33) ma-qri-t

ћetta ktab

'I didn't read any book.'

23 Elabbas Benmamoun, op. cit., p. 269. 


\section{(33’) *ma-qrit-šs $\quad$ hetta ktab \\ 'I didn't read any book.'}

La négation est une unité à portée, c'est-à-dire une unité exerçant " un contrôle structurel sur un fragment de la phrase ${ }^{24}$ ». Ce fragment peut être la relation prédicative ou un seul terme de la relation ${ }^{25}$. Pour illustrer ce point, nous opposerons dans ce qui suit des énoncés non négatifs à des énoncés négatifs.

En AM, dans les énoncés assertifs non négatifs, les noms denses (non comptables, compacts) sont déterminés par l'article défini (34) et les noms discontinus, discrets sont déterminés (quantifiés) par ce que Philippe Marçais ${ }^{26}$ définit comme «le degré zéro de la détermination » (35):

(34) $\hbar m e d ~ \check{s} r a-\varnothing \quad l-\hbar l i b$

Ahmed acheter-il.perf le-lait

'Ahmed a acheté du lait.'

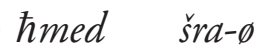 $\emptyset-k t a b$
Ahmed acheter-il.perf livre
'Ahmed a acheté un livre.'

Dans les énoncés assertifs négatifs, lorsque la négation porte sur la relation prédicative, elle est réalisée par l'association de $m a$ - et $-s$ dans le contexte d'un nom déterminé par le "défini » (36 et 37), mais par le seul ma-dans le contexte d'un nom quantifié par le "degré zéro de la détermination " (38 et 39) :

(36) $m a-\varepsilon e n d-u-s ̌ \quad l-f l u s$

nég-chez-lui-q le-argent

'Il n'a pas d'/l'argent.'

(37) $m a-\varepsilon e n d-u-s ̌ r \quad l-k t a b$

nég-chez-lui-q le-livre

'Il n'a pas le livre.'

24 cf. note 17 supra : Henning Nølke, Linguistique modulaire : de la forme au sens, Louvain-Paris, Peeters, 1994, p. 228.

25 Nous nous appuyons ici sur Philippe Marçais, op.cit. et Dominique Caubet, La détermination en arabe marocain, Paris, Université Paris 7, 1983.

26 Philippe Marçais, op. cit., p. 395. 
(38) $m a-\varepsilon e n d-u \quad \emptyset-f l u s$ nég-chez-lui argent 'Il n'a pas d'argent.'

(39) $m a-\varepsilon e n d-u \quad \emptyset-k t a b$ nég-chez lui livre 'Il n'a pas de livre."

Lorsque la négation porte sur un terme de la relation prédicative (et non sur toute la relation), l'association entre $m a$ - et- $r$ est réalisée quelle que soit la nature de la détermination nominale : (39) $\begin{array}{llll}\text { ma-eend-u-s } & \text { flus } & \text { cend-u } & \text { l-mlayar } \\ \text { nég-chez-lui-q argent chez-lui } & \text { le-million.pl }\end{array}$ 'Il n'a pas de l'argent, il a (possède) des millions'. (Ce n'est pas de l'argent qu'il a ! Ce sont des millions!) (40) ktab šra-ø mažella nég-acheter-il.perf-q livre acheter-il.perf magazine 'Il n'a pas acheté de livre, il a acheté un magazine'.

En (39) et (40), les noms flus « argent " et ktab « livre », bien que non déterminés par le défini, ne sont pas vagues, ne sont pas "non définis ". Au contraire, ils sont spécifiés et définis parce que marqués par une intonation forte ou une pause audible. Ce procédé de focalisation permet à un locuteur/énonciateur de marquer explicitement le foyer de son énonciation. Le processus d'identification du focus est nécessaire au processus d'interpréta$\operatorname{tion}^{27}$. Considérons les exemples (41) et (42) ci-dessous :

(41) Erad-na-hum kull-hum l-l-Eers $u$ wahedma-ža- $\emptyset$ inviter-nous.perf-eux tout-eux à-le-mariage et un (=numéral) nég-venir-il.perf

'Nous les avons tous invités au mariage et aucun n'est venu.'

(42) crad-na-hum kull-hum l-l-eers u wahedmaža-ø-š inviter-nous.perf-eux tout-eux à-le-mariage et un nég-venir-il. perf-q 'Nous les avons tous invités au mariage et un (seul) n'est pas venu.'

27 Henning Nølke, op. cit., 1994, p. 194. 
En (41), le quantifieur wahed " un » s'associe à la négation $m a$ - pour nier la relation prédicative. Dans ce contexte, wahed signifie " un X quelconque, non défini », l'élément $-s ̌$ n'est donc pas nécessaire pour satisfaire l'association négative. En (42), wahed ne fonctionne plus comme le deuxième élément de l'association négative, c'est-à-dire comme un quantifieur indéfini, mais signifie " un X précis, spécifié, défini »; l'association avec $-\check{s}$ est alors nécessaire pour réaliser l'association négative. En (42), wahed est le focus de la négation.

Par ailleurs, en arabe marocain - tout comme en français - le locuteur/énonciateur peut recourir à un autre type de procédé de focalisation : les focalisateurs que Nølke analyse comme des adverbes paradigmatisants : "Un adverbe paradigmatisant introduit en tant que présupposé un paradigme d'éléments semblables à l'élément auquel il est attaché dans la phrase actuelle ${ }^{28}$ ». Ces adverbes évoquent un paradigme d'éléments alternatifs sur lesquels ils agissent sémantiquement et avec lesquels ils sont syntaxiquement liés. En AM, les unités yir 'seulement' et $\hbar$ etta " même, jusqu'à » fonctionnent comme des adverbes paradigmatisants, c'est-à-dire comme des focalisateurs, en particulier lorsqu'ils déterminent un nom ${ }^{29}$. On s'arrêtera ici sur l'unité $\hbar$ etta, que l'on peut trouver déterminant un nom dans des énoncés non négatifs :

$\begin{array}{llll}\text { hetta } \quad l \text {-mra } & \text { ža-t } & l \text {-l-Eers } \\ \text { même } & \text { la-femme } & \text { venir-elle.perf } & \text { à-le-mariage } \\ \text { 'Même la femme est venue au mariage.' }\end{array}$

(44) $\hbar$ etta

mra

$\check{z} a-t$

l-l-eers

même

q.femme

venir-elle.perf à-le-mariage

'Même une femme est venue au mariage.'

$\overline{28}$ Henning Nølke, Les adverbes paradigmatisants : fonction et analyse, Copenhague, Akademisk Forlag, 1983, p. 19.

29 Jamal Ouhalla, "The Structure and Logical Form of Negative Sentences ", dans Jamal Ouhalla et Ur Shlonsky (dir.), Themes in Arabic and Hebrew syntax, Netherlands,Kluwer, 2002, p. 18, note 2. 
Et dans des énoncés négatifs (45-46) :
(45) Ћetta ø-mra
$m a-\check{z} a-t$
$l-l$-eers
même q-femme nég-venir-elle.perf à-le-mariage

Lit. : même une (quelconque) femme n'est pas venue au mariage. 'Aucune femme n'est venue au mariage.'

\section{(46) hetta ši-mra ma ža-t \\ l-l-Eers \\ même q-femme neg-venir-elle.perf à-le-mariage}

Lit. : même certaine (trace de) femme n'est pas venue au mariage. '(Absolument) Aucune femme n'est venue au mariage.'

Le nom mra «femme " est déterminé par « le degré zéro de la détermination " en (45) et par le quantifieur indéfini ši en (46). Dans ces deux exemples, la négation porte sur la relation prédicative et hetta marque le nom mra comme étant le focus de la relation. Il introduit le présupposé qu'aucun membre du paradigme (ensemble d'éléments semblables auquel appartient mra et duquel il a été prélevé) pas même " une femme quelconque » ne valide la relation prédicative, "n'est venue au mariage ". (47)
hetta $\emptyset$-mra
ma $\check{z} a-t-\check{s}$
$l-l$-eers
même q.femme neg-venir-elle.perf-q 'Pas une femme n'est venue au mariage.'
hetta l-mra
$m a \check{z} a-t-\grave{s}$
l-l-eers
même la-femme nég-venir-elle.perf-q à-le-mariage
'Même la femme n'est pas venue au mariage.

Ici, le nom mra est présenté comme une entité définie, par l'entremise du déterminant en (48) et d'une intonation marquée en (47); de ce fait, l'association de $m a$ - et $-s ̌$ est nécessaire pour satisfaire l'association négative. Le focalisateur $\hbar$ etta marque $m r a$ comme le focus de la relation et introduit la présupposition que tous les membres du paradigme auquel appartient $m r a$ ne sont pas venus, et particulièrement 'une femme bien identifiée, bien connue'. 
Considérons pour finir les exemples (49) et (50) ci-dessous :
(49) hetta nhar
$m a-\check{z} a-\emptyset$
l-d-dar
même jour
nég-venir-il.perf
à-la-maison

Lit. : même un jour (quelconque), il n'est pas venu à la maison. 'Il n'y a pas un jour où il ne soit venu à la maison.'
(50) $\hbar$ etta
nhar
$m a-\check{z} a-\emptyset-\check{s}$
l-d-dar
même
jour
nég-venir-il.perf-q
à-déf-maison

Lit. : même un jour/pas un jour, il n'est pas venu à la maison. 'Il n'y a pas un jour où il ne soit pas venu à la maison.'

En (49), la négation porte sur la relation prédicative <lui venir maison $>$. La relation n'est validée pour aucun des éléments $\mathrm{du}$ paradigme auquel appartient nhar 'jour', même pas pour " un jour quelconque non nul ». Le degré zéro de la détermination a valeur ici d'indéfini. Du fait de l'association négative, le syntagme hetta nhar fait partie de la structure argumentale du verbe, entrant ainsi sous la portée de la négation. Il est d'ailleurs possible de le déplacer (voir 49' et 49") :
(49') $m a-\check{z} a-\varnothing$
ћetta nhar
l-d-dar
nég-venir-il.perf même jour
à-la-maison
'Il n'est venu aucun jour à la maison.'
(49") $m a-\check{z} a-\varnothing$
l-d-dar
ћetta nhar
nég-venir-il.perf
à-la-maison
même jour
'Il n'est venu aucun jour à la maison.'

En revanche, cela est beaucoup moins aisé pour l'exemple (50), le syntagme hetta nhar devant obligatoirement occuper les positions liminaires de la phrase :
(50’) ћetta
nhar
$m a-\check{z} a-\emptyset-\grave{s}$
l-d-dar

'Il n'y a pas un jour où il ne soit pas venu à la maison.'

\section{(50") $m a-z ̌ a-\emptyset-s \quad$ l-d-dar hetta nhar \\ 'Il n'est pas venu à la maison. Pas un jour.'}

Dans ces contextes, hetta nhar est prédicatif. De ce fait, il échappe à la portée de la négation, ce qui permet l'association entre ma- et -řs. L'exemple (50) n'est pas une assertion directe mais 
une assertion adversative : le locuteur réfute un énoncé positif émis par un locuteur antérieur, qui aurait dit : Il y a un jour où il n'est pas venu à la maison, et énonce Je dis qu'il n'y a pas un seul jour (même un jour quelconque) où il ne soit pas venu à la maison ${ }^{30}$.

\section{Conclusion}

La négation grammaticale en arabe marocain est un opérateur syntactico-sémantique complexe; sa description nécessite d'adopter conjointement plusieurs approches. En tant qu'opérateur, la négation exerce un contrôle structurel sur son contexte et entre dans un réseau de relations complexes avec les autres catégories grammaticales, mais aussi avec d'autres domaines fonctionnels tels la quantification. Le temps nécessaire au traitement de l'information, à l'interprétation des énoncés négatifs est plus long que celui nécessaire aux énoncés non négatifs du fait de ces interactions. Le degré de complexité varie toutefois avec les variations de portée de la négation.

$\overline{30}$ Oswald Ducrot, Le dire et le dit, Paris, Minuit, 1984. 


\section{Bibliographie}

Benmamoun, Elabbas, "Licensing of Polarity Items in Moroccan Arabic ", Natural Language and Linguistics Theory, $\mathrm{n}^{\circ}$ 15, 1997, p. 263-287.

Benmamoun, Elabbas, The Feature Structure of Functional Categories. A Comparative Study of Arabic Dialects, New-York/Oxford, Oxford University Press, 2000.

Benmamoun, Elabbas, "Licensing Configurations: The Puzzle of Head Negative Polarity Items ", Linguistic Inquiry, vol. 33, n 1, 2006, p. 141-149.

Brustad, Kristen E., The Syntax of Spoken Arabic. A Comparative Study of Moroccan, Egyptian, Syrian, and Kuwaiti Dialects, Washington D.C., Georgetown University Press, 2000.

Caubet, Dominique, La détermination en arabe marocain, Paris, Université Paris 7, 1983.

Caubet, Dominique et Salem Chaker (dir.), La négation en berbère et en arabe maghrébin, Paris, L'Harmattan, 1996.

Chatar-Moumni, Nizha, "Quelques aspects du fonctionnement de la négation en arabe marocain ", La linguistique, vol. 44, $\mathrm{n}^{\circ}$ 2, 2008, p. 81-97.

Chatar-Moumni, Nizha, "Negation in Moroccan Arabic: Scope and Focus », dans Reem Bassiouney et Graham Katz (dir.), Arabic Language and Linguistics, Georgetown, Georgetown University Press, 2012, p. 3-15.

Dahl, Östen, « Typology of Sentence Negation », Linguistics, vol. 17, 1979, p. 79-106.

Dixon, Robert, The Rise and Fall of Languages, Cambridge, Cambridge University Press, 1997.

Ducrot, Oswald, Le dire et le dit, Paris, Minuit, 1984.

Fruyt, Michèle, "Négation et grammaticalisation en latin ", Revue de linguistique latine du centre Alfred Ernout : de lingua latina, nº 1, 2008, (revue électronique : http://www.fabula.org/actualites/de-lingualatina-revue-de-linguistique-latine-du-centre-alfred-ernout_28051. php ), article consultable à : http://www.paris-sorbonne.fr/IMG/pdf/ Fruyt2.pdf (visité le 15/11/2013).

Harrell, Richard Slade, A Short Reference Grammar of Moroccan Arabic, Washington DC, Georgetown University Press, 1962.

Hoyt, Frederick, "Sentential Negation Marking in Palestinian and Moroccan Arabic. A Study in Comparative Syntax ", Seminar of Arabic 
dialectology, http://fmhoyt.colliertech.org/Hoyt(05)_NegationInTwoDialects OfArabic.pdf (consulté le 15/11/2013), 2005.

Jespersen, Otto, « Negation in English and Other Languages », Copenhagen, Høst, 1917.

Lameen Souag, Mostafa, Explorations in the Syntactic Cartography of Algerian Arabic, thèse SOAS, London University, 2006.

Lucas, Christopher, "Jespersen's Cycle in Arabic and Berber ", Transactions of the Philological Society, Cambridge, Cambridge University, vol. 105, $\mathrm{n}^{\circ}$ 3, 2007, p. 398-431.

Lucas, Christopher et Elliott Lash, "Contact as Catalyst: The Case for Coptic Influence in the Development of Arabic Negation ", Journal of Linguistics, $\mathrm{n}^{\circ} 46,2010$, p. 379-413.

Marçais, Philippe, Le parler arabe de Djidjelli, Paris, Maisonneuve, 1953.

Meillet, Antoine, "L'évolution des formes grammaticales ", Scientia. Revue internationale de synthèse scientifique, vol. 12, $\mathrm{n}^{\circ}$ XXVI-6, 1912, p. 384-400.

Miestamo, Matti, "On the Complexity of Standard Negation ", dans Mickael Suominen et al. (dir.), A Man of Measure: Festschrift in Honour of Fred Karlsson on his 60th Birthday, Turku, Linguistic Association of Finland, 2006, p. 345-356.

Muller, Claude, "L'association négative ", Langue française, vol. 62, $\mathrm{n}^{\circ} 1$, 1984, p. 59-94.

Nølke Henning, Les adverbes paradigmatisants : fonction et analyse, Copenhague, Akademisk Forlag, 1983.

Nølke, Henning, Linguistique modulaire : de la forme au sens, Louvain, Paris, Peeters, 1994.

Nølke, Henning, "Les lectures de ne... pas : éléments d'une explication modulaire ", Linx [En ligne], 5, 1994, mis en ligne le 18 juillet 2012, consulté le 25 octobre 2012. URL : http://linx.revues.org/1212, DOI : 10.4000/linx.1212.

Ouhalla, Jamal, "The Structure and Logical Form of Negative Sentences ", dans Jamal Ouhalla et Ur Shlonsky (dir.), Themes in Arabic and Hebrew Syntax, Netherlands, Kluwer, 2002, p. 299-320.

Reschler, Nicolas, Complexity: A Philosophical Overview, New Jersey, Transaction publishers, 1998. 